\title{
ON THE MEAN VALUE PROPERTY OF HARMONIC FUNCTIONS AND BEST HARMONIC $L^{1}$-APPROXIMATION
}

\author{
MYRON GOLDSTEIN, WERNER HAUSSMANN AND LOTHAR ROGGE
}

\begin{abstract}
The present paper deals with the inverse mean value property of harmonic functions and with the existence, uniqueness, and characterization of a best harmonic $L^{1}$-approximant to strictly subharmonic functions. The main theorem concerning the inverse mean value property of harmonic functions is based on a generalization of a theorem due to $\ddot{U}$. Kuran as well as on an approximation theorem proved by J. C. Polking and also by L. I. Hedberg. The inverse mean value property will be applied in order to prove necessary and sufficient conditions for the existence of a best harmonic $L^{1}$-approximant to a subharmonic function $s$ satisfying $\Delta s>0$ a.e. in the unit ball.
\end{abstract}

1. Introduction and main results. Throughout the paper let $2 \leq n \in \mathbf{N}$, and let $B:=\left\{x \in \mathbf{R}^{n}:|x|<1\right\}$ be the open unit ball, and $\bar{B}$ its closure. Denote by $\mathscr{H}(B)$ the space of all real-valued functions on $\bar{B}$ which are harmonic in $B$. Let $\mathscr{C}(\bar{B})$ be the space of all real-valued continuous functions on $\bar{B}$, and $\mathscr{C}^{2}(B)$ the space of all real-valued functions on $\bar{B}$ which are twice continuously differentiable on $B$. In addition, let $B_{0}:=\left\{x \in \mathbf{R}^{n}:|x|<1 / \sqrt[n]{2}\right\}$, and denote by $\Delta$ the Laplacian differential operator. For a subset $E \subset \mathbf{R}^{n}$, let $E^{\circ}$ denote the interior of $E$.

Given a subharmonic function $s \in \mathscr{C}(\bar{B})$, we ask for a best harmonic $L^{1}$-approximant $h^{*} \in \mathscr{H}(B) \cap \mathscr{C}(\bar{B})$ to $s$, i.e. a function $h^{*} \in \mathscr{H}(B) \cap \mathscr{C}(\bar{B})$ which satisfies

$$
\left\|s-h^{*}\right\|_{1} \leq\|s-h\|_{1} \quad \text { for all } h \in \mathscr{H}(B) \cap \mathscr{C}(\bar{B}),
$$

where $\|\cdot\|_{1}$ denotes the $L^{1}$-norm defined by

$$
\|v\|_{1}:=\frac{1}{\lambda(\bar{B})} \int_{\bar{B}}|v| d \lambda \quad\left(v \in L^{1}(\bar{B})\right)
$$

with the $n$-dimensional Lebesgue measure $\lambda$. For brevity we shall also write $\int v$ instead of $\int v d \lambda$.

It is the topic of this paper to investigate the questions of existence and uniqueness of a best harmonic $L^{1}$-approximant as well as its characterization in terms of boundary value (or interpolation) conditions of Dirichlet type. In addition we shall deal with the inverse mean value property of harmonic functions.

The following two theorems are the main results of this paper.

Received by the editors January $5,1986$.

1980 Mathematics Subject Classification (1985 Revision). Primary 31B05, 41A63, 41A50; Secondary 31B20.

Key words and phrases. Harmonic and subharmonic functions, inverse mean value property, harmonic $L^{1}$-approximation.

Research of the first author supported in part by the SERC Grant GR/D/17731. 
THEOREM 1. Let $D \subset \mathbf{R}^{n}$ be a nonvoid bounded open set such that $(\alpha) \mathbf{R}^{n} \backslash \bar{D}$ is connected.

Suppose there exists a point $x_{0} \in \mathbf{R}^{n}$ such that for every function $h$ harmonic in $\mathbf{R}^{n}$

$$
h\left(x_{0}\right)=\frac{1}{\lambda(D)} \int_{D} h
$$

or

$$
h\left(x_{0}\right)=\frac{1}{\lambda(\bar{D})} \int_{\bar{D}} h
$$

Then $\bar{D}$ is a closed ball centered at $x_{0}$.

Trivial examples show that $D$ itself does not need to be an open ball and that $(\alpha)$ cannot be replaced by " $\mathrm{R}^{n} \backslash D$ is connected."

This theorem will be the main tool in the proof of the following approximation theorem.

THEOREM 2. Let $s \in \mathscr{C}^{2}(B) \cap \mathscr{C}(\bar{B})$ with $\Delta s>0$ a.e. in $B$. A function $h^{*} \in \mathscr{H}(B) \cap \mathscr{C}(\bar{B})$ is a best harmonic $L^{1}$-approximant to $s$ if and only if $h^{*}$ satisfies the following two conditions:

(i) $\left.h^{*}\right|_{\partial B_{0}}=\left.s\right|_{\partial B_{0}}$,

(ii) $s-h^{*}>0$ a.e. on $\bar{B} \backslash \bar{B}_{0}$.

In addition, if (i) and (ii) are satisfied, then $h^{*}$ is the unique best $L^{1}$-approximant to $s$.

We remark that the set $\partial B_{0}$ for which we have to solve the Dirichlet problem does not depend on the particular function $s$ to be approximated.

The proofs of Theorems 1 and 2 will be given in $\S \S 2$ and 3, respectively. In order to prove Theorem 1 we first point out a variant and a generalization of Kuran's theorem [15] concerning the mean value property of harmonic functions (Theorem 3). Then, with the aid of an approximation theorem due to Hedberg [13] and Polking [17], we get the proof of Theorem 1. In $\S 3$, we first point out the sufficiency of conditions (i) and (ii) of Theorem 2 even under weaker hypotheses. For the necessity of the conditions we need a result concerning zeros of subharmonic functions (Lemma 6). Then Theorem 1 will be applied to complete the proof of Theorem 2. Finally, in $\S 4$, we shall show that there are subharmonic functions in $\mathscr{C}^{2}(B) \cap \mathscr{C}(\bar{B})$ satisfying $\Delta s>0$ a.e. in $B$ which do not have a best harmonic $L^{1}$-approximant in $\mathscr{H}(B) \cap \mathscr{C}(\bar{B})$.

Throughout this paper, let $\omega_{n}$ denote the volume of the $n$-dimensional unit ball. Furthermore, given a set $D \subset \mathbf{R}^{n}$ and a function $u \in \mathscr{C}(D)$, by $Z(u)$ we denote the set of all zeros of $u$ in $D$. Also we shall use the $L^{p}$-norm defined by

$$
\|v\|_{p, D}:=\|v\|_{p}:=\left(\frac{1}{\lambda(D)} \int_{D}|v|^{p} d \lambda\right)^{1 / p}
$$

for $1 \leq p<\infty$, where $D \subset \mathbf{R}^{n}$ is a set of positive and finite Lebesgue measure, and $v \in L^{\underline{p}}(D)$.

We refer to Gilbarg and Trudinger [9] and Hayman and Kennedy [11] concerning harmonic and subharmonic functions as well as to Cheney [5] and Lorentz [16] for general results on $L^{1}$-approximation. 
2. Inverse mean value property of harmonic functions. In order to prove Theorem 1, we first show a variant and a generalization of Kuran's [15] inverse mean value theorem for harmonic functions. We shall adopt the following notation:

Given a closed set $F \subset \mathbf{R}^{n}$, define the function spáce $h(F)$ to consist of all functions which are harmonic in some neighborhood of $F$. The subspace of $L^{p}(F)$ consisting of all functions harmonic in $F^{\circ}$ will be denoted by $h_{p}(F)$.

THEOREM 3. Let $D$ be an open set of finite Lebesgue measure in the Euclidean space $\mathbf{R}^{n}$, and let $p$ be a fixed real number satisfying $1 \leq p<n /(n-1)$.

(A) Suppose that there exists an interior point $x_{0}$ of $\bar{D}$ such that for every function $h \in h_{p}(\bar{D})$

$$
h\left(x_{0}\right)=\frac{1}{\lambda(D)} \int_{D} h
$$

or

$$
h\left(x_{0}\right)=\frac{1}{\lambda(\bar{D})} \int_{\bar{D}} h .
$$

Then $\bar{D}$ is a closed ball centered at $x_{0}$.

(B) If for a given $x_{0} \in D$ equation (2) holds for every function $h$ harmonic in $D$ and $L^{p}$-integrable over $D$, then $D$ is an open ball centered at $x_{0}$.

REMARK. In [10, p. 258] our result (B) was established for the case $p=1$ based on a theorem of Kuran [15] who had considered the case where $p=1$ and $D$ a domain (= open connected set). A close examination of Kuran's proof shows that in order to prove (A) and (B) one does not need all $L^{1}$-integrable harmonic functions but only those lying in $h_{p}(\bar{D})$, resp. being harmonic in $D$, and $L^{p}$-integrable over $D$ as "test functions" for some $p \in[1, n /(n-1)[$.

The proofs of (A) and (B) follow the same line so that we shall point out (A) and add in $[\cdots]$ those minor changes which have to be made in order to prove (B). This will be demonstrated in the following.

ProOF OF THEOREM 3. First we follow the proof by Kuran: Determine a point $x_{1} \in \mathbf{R}^{n} \backslash \bar{D}^{\circ}$ [resp. $\mathbf{R}^{n} \backslash D$ ] such that

$$
0<\left|x_{1}-x_{0}\right| \leq\left|x-x_{0}\right| \text { for all } x \in \mathbf{R}^{n} \backslash \bar{D}^{\circ}\left[\text { resp. } \mathbf{R}^{n} \backslash D\right] \text {. }
$$

Define $E$ to be the open ball with center $x_{0}$ and radius $r:=\left|x_{1}-x_{0}\right|$, hence $\bar{E} \subset \bar{D}$ [resp. $E \subset D$ ]. It now suffices to show that $D \subset \bar{E}$, since this implies $\bar{D} \subset \bar{E}$ [resp. $D \subset E$ ] and hence $\bar{E}=\bar{D}$ [resp. $E=D$ ]. Assume that $D \backslash \bar{E} \neq \varnothing$. Then $\lambda(D \backslash \bar{E})>0$. The function $h$ defined on $\mathbf{R}^{n} \backslash\left\{x_{1}\right\}$ by

$$
h(x):=r^{n-2}\left(\left|x-x_{0}\right|^{2}-r^{2}\right)\left|x-x_{1}\right|^{-n}+1=: r^{n-2} \cdot K(x)+1
$$

is harmonic in $\mathbf{R}^{n} \backslash\left\{x_{1}\right\}$ (since $K$ is proportional to the Poisson kernel of $E$ and of $\mathbf{R}^{n} \backslash \bar{E}$ at $\left.x_{1}\right)$. In particular, $h$ is harmonic in $\bar{D}^{\circ}$ [resp. $D$ ]. In addition, $h\left(x_{0}\right)=0$ and $h(x) \geq 1$ outside of $\bar{E}$. Furthermore, $h$ is $L^{p}$-integrable in $\bar{D}$ [resp. $D$ ] since $|K(x)|$ behaves like $\left|x-x_{1}\right|^{1-n}$ in some neighborhood $N$ of $x_{1}$, i.e. $|K(x)|^{p}$ behaves like $\left|x-x_{1}\right|^{(1-n) p}$ with

$$
(1-n) p>(1-n) \cdot n /(n-1)=-n .
$$


In addition, $|h|$ is bounded in $D \backslash N$. Therefore, if (2) is satisfied, then

$$
0=\int_{D} h=\int_{E} h+\int_{D \backslash E} h=\int_{D \backslash \bar{E}} h \geq \int_{D \backslash \bar{E}} 1>0,
$$

a contradiction. Hence $\bar{E}=\bar{D}$ [resp. $E=D$ ]. If $\left(2^{\prime}\right)$ is fulfilled, then the assumption $D \backslash \bar{E} \neq \varnothing$ leads to

$$
0=\int_{\bar{D}} h=\int_{E} h+\int_{\bar{D} \backslash E} h=\int_{\bar{D} \backslash \bar{E}} h \geq \int_{\bar{D} \backslash \bar{E}} 1>0,
$$

a contradiction, too, hence $\bar{E}=\bar{D}$.

Our proof of Theorem 1 is based on a result by Hedberg [13] and Polking [17] as well as on a theorem by Brelot [3] and Deny [6] (see also the theorems of WalshLebesgue and Runge type in Gamelin [7, p. 28] and in [8, Theorem 1]).

ProOF OF THEOREM 1. Put $F:=\bar{D} \cup\left\{x_{0}\right\}$ and let $g \in h(F) . F$ is compact, and its complement is connected by $(\alpha)$. Hence by an approximation theorem due to Brelot [3] and Deny [6] (see also Gamelin [7, p. 28] for $n=2$, and [8, Theorem 1] for $n \geq 3)$ it follows that for each $\varepsilon>0$ there exists a function $\omega \in \mathscr{H}\left(\mathbf{R}^{n}\right)$ with

$$
\|g-\omega\|_{\infty, F}:=\sup _{x \in F}|g(x)-\omega(x)|<\varepsilon .
$$

Since $x_{0} \in F$ and $\lambda(\bar{D})<\infty$, by (1), resp. $\left(1^{\prime}\right)$, we obtain

$$
g\left(x_{0}\right)=\frac{1}{\lambda(D)} \int_{D} g, \quad \text { resp. } g\left(x_{0}\right)=\frac{1}{\lambda(\bar{D})} \int_{\bar{D}} g .
$$

Now we shall show that $x_{0} \in \bar{D}^{\circ}$, hence $F=\bar{D}$. Assume indirectly $x_{0} \notin \bar{D}^{\circ}$. Then there exists a sequence $\left(x_{j}\right)_{j \in \mathbf{N}}$ in $\mathbf{R}^{n} \backslash \bar{D}$ with

$$
0<\left|x_{j}-x_{0}\right| \rightarrow 0 \quad(\text { for } j \rightarrow \infty) \text {. }
$$

The functions $\varphi_{j}^{(n)}$ defined by

$$
\varphi_{j}^{(n)}(x):= \begin{cases}\frac{\log \left|x-x_{j}\right|}{\log \left|x_{0}-x_{j}\right|} & \text { for } n=2, \\ \frac{\left|x_{0}-x_{j}\right|^{n-2}}{\left|x-x_{j}\right|^{n-2}} & \text { for } n \geq 3\end{cases}
$$

are harmonic in a neighborhood of $F$. Hence, by (3) we have

$$
1=\varphi_{j}^{(n)}\left(x_{0}\right)=\frac{1}{\lambda(D)} \int_{D} \varphi_{j}^{(n)}, \quad \text { resp. } 1=\frac{1}{\lambda(\bar{D})} \int_{\bar{D}} \varphi_{j}^{(n)} .
$$

On the other hand, for either of the integrals occurring in (4) and for a sufficiently large $R$ (independent of $j$ ) we have

$$
\begin{aligned}
\left.\mid \begin{array}{l}
\int_{D} \varphi_{j}^{(n)} \mid \\
\int_{\bar{D}} \varphi_{j}^{(n)}
\end{array}\right\} & \leq \int_{\bar{D}}\left|\varphi_{j}^{(n)}\right|=\left\{\begin{array}{l}
\left|\frac{1}{\log \left|x_{0}-x_{j}\right|}\right| \cdot \int_{\bar{D}}|\log | x-x_{j}|| \text { for } n=2, \\
\left|x_{0}-x_{j}\right|^{n-2} \cdot \int_{\bar{D}} \frac{1}{\left|x-x_{j}\right|^{n-2}} \quad \text { for } n \geq 3,
\end{array}\right. \\
& \leq\left\{\begin{array}{l}
\left|\frac{1}{\log \left|x_{0}-x_{j}\right|}\right| \cdot \int_{0}^{R}|\log r| \cdot r d r \\
\left|x_{0}-x_{j}\right|^{n-2} \cdot \int_{0}^{R} r^{2-n} \cdot r^{n-1} d r
\end{array} \rightarrow 0\right.
\end{aligned}
$$


(for $j \rightarrow \infty$ ), which yields a contradiction to (4). Thus $x_{0} \in \bar{D}^{\circ}$, hence $F=\bar{D}$.

Now let $1<p<n /(n-1)$ be fixed. Then $n<q:=p /(p-1)<\infty$. As $\bar{D}$ is compact, we obtain from Hedberg [13, Lemma 1 and Theorem 3] (see also Polking [17]) that $h(\bar{D})$ is $L^{p}(\bar{D})$-dense in $h_{p}(\bar{D})$. Hence for each $f \in h_{p}(\bar{D})$ there exists a sequence of functions $g_{j} \in h(\bar{D})$ with

$$
\left\|f-g_{j}\right\|_{1, \bar{D}} \leq\left\|f-g_{j}\right\|_{p, \bar{D}} \rightarrow 0 .
$$

Therefore, by the mean value property of harmonic functions (with respect to a ball $K \subset \bar{D}^{\circ}$ centered at $x_{0}$ ) we get

$$
\lim _{j \rightarrow \infty} g_{j}\left(x_{0}\right)=f\left(x_{0}\right) .
$$

Now, by (3) and (5), we obtain for each $f \in h_{p}(\bar{D})$

$$
f\left(x_{0}\right)=\lim _{j \rightarrow \infty} g_{j}\left(x_{0}\right) \stackrel{(3)}{=} \lim _{j \rightarrow \infty} \frac{1}{\lambda(D)} \int_{D} g_{j} \stackrel{(5)}{=} \frac{1}{\lambda(D)} \int_{D} f,
$$

respectively

$$
f\left(x_{0}\right)=\lim _{j \rightarrow \infty} g_{j}\left(x_{0}\right) \stackrel{(3)}{=} \lim _{j \rightarrow \infty} \frac{1}{\lambda(\bar{D})} \int_{\bar{D}} g_{j} \stackrel{(5)}{=} \frac{1}{\lambda(\bar{D})} \int_{\bar{D}} f .
$$

Using (6), resp. $\left(6^{\prime}\right)$, the assertion follows from Theorem $3(\mathrm{~A})$.

3. Proof of the Approximation Theorem 2. In this section we first show that conditions (i) and (ii) of Theorem 2 imply that $h^{*}$ is a best harmonic $L^{1}$ approximant to $s$. In order to prove this, we use the following result which can be found in Singer's book [19, p. 46].

Proposition 4. Let $V \subset \mathscr{C}(\bar{B})$ be a vector space, $s \in \mathscr{C}(\bar{B}) \backslash V$, and $h^{*} \in V$. Then we have the following equivalence:

(a) $h^{*}$ is a best $L^{1}$-approximant to $s$;

(b) there exists a Lebesgue measurable function $\alpha$ on $Z\left(s-h^{*}\right)$ such that

$\left(\mathrm{b}_{1}\right)|\alpha(t)| \leq 1$ a.e. on $Z\left(s-h^{*}\right)$;

$\left(\mathrm{b}_{2}\right) \int_{Z\left(s-h^{*}\right)} \alpha h+\int_{\bar{B} \backslash Z\left(s-h^{*}\right)} h \operatorname{sign}\left(s-h^{*}\right)=0$ for all $h \in V$.

With the aid of this proposition we can prove the sufficiency of conditions (i) and (ii) of Theorem 2 even under weaker assumptions:

THEOREM 5. Let $s \in \mathscr{C}(\bar{B})$ be subharmonic in $B$ and suppose that $h^{*} \in$ $\mathscr{H}(B) \cap \mathscr{C}(\bar{B})$ satisfies

(i) $\left.h^{*}\right|_{\partial B_{0}}=\left.s\right|_{\partial B_{0}}$,

(ii) $s-h^{*}>0$ a.e. on $\bar{B} \backslash \bar{B}_{0}$.

Then $h^{*}$ is the unique best harmonic $L^{1}$-approximant to $s$.

PROOF. Since $s-h^{*}$ is subharmonic and $\left.\left(s-h^{*}\right)\right|_{\partial B_{0}}=0$, it follows that $s-h^{*} \leq 0$ in $\bar{B}_{0}$. Define the function $\alpha$ (according to Proposition 4 ) by

$$
\alpha(t):=-1 \text { for } t \in Z\left(s-h^{*}\right) .
$$

Then we have for any $h \in \mathscr{H}(B) \cap \mathscr{C}(\bar{B})$

$$
\int_{Z\left(s-h^{*}\right)} \alpha h+\int_{\bar{B} \backslash Z\left(s-h^{*}\right)} h \operatorname{sign}\left(s-h^{*}\right)=\int_{\bar{B}_{\bar{B}} \bar{B}_{0}} h-\int_{\bar{B}_{0}} h=0
$$


since, by the mean value property for harmonic functions, we have

$$
\frac{1}{2} \omega_{n} h(0)=\int_{\bar{B}_{0}} h=\frac{1}{2} \int_{\bar{B}} h=\int_{\bar{B} \backslash \bar{B}_{0}} h .
$$

Now, by (7) and Proposition $4, h^{*}$ is a best $L^{1}$-approximant to $S$.

Next we shall prove the uniqueness of $h^{*}$. Suppose $h^{\prime}$ is another best harmonic $L^{1}$-approximant. Then, using the continuity of $s-h^{*}$ and $s-h^{\prime}$ on $\bar{B}$, we get (see Rice $[18$, p. 107] or $[10$, p. 260])

$$
\left(s-h^{*}\right) \cdot\left(s-h^{\prime}\right) \geq 0 \text { on } \bar{B} .
$$

We distinguish two cases:

( $\alpha$ ) If $s-h^{*}<0$ in $B_{0}$, then $s-h^{\prime} \leq 0$ in $B_{0}$ as well. From (ii) and (9) we conclude $s-h^{\prime} \geq 0$ a.e. and hence, by continuity, everywhere in $\bar{B} \backslash \bar{B}_{0}$; thus $\left.\left(s-h^{\prime}\right)\right|_{\partial B_{0}}=0$. By (i) and the uniqueness of the solution of the Dirichlet problem for $s$ with respect to $\partial B_{0}$ we get $h^{\prime}=h^{*}$ on $\bar{B}_{0}$ and hence on $\bar{B}$.

$(\beta)$ If there is an $x_{1} \in B_{0}$ such that $\left(s-h^{*}\right)\left(x_{1}\right)=0$, then, by the maximum principle, $\left.\left(s-h^{*}\right)\right|_{\bar{B}_{0}}=0$. Again, by (ii) and (9), we get $s-h^{\prime} \geq 0$ on $\partial B_{0}$. Hence $h^{*} \geq h^{\prime}$ on $\partial B_{0}$. If $h^{*}=h^{\prime}$ on $\partial B_{0}$, then we obtain $h^{*}=h^{\prime}$ on $\bar{B}$. Otherwise $h^{*}>h^{\prime}$ on $B_{0}$ by the minimum principle, hence

$$
s-h^{\prime}>s-h^{*}=0 \text { on } B_{0},
$$

and there exists an $x_{0} \in \partial B_{0}$ with $h^{*}\left(x_{0}\right)>h^{\prime}\left(x_{0}\right)$, i.e. $\left(s-h^{\prime}\right)\left(x_{0}\right)>0$. Therefore, by continuity, there exists a ball $K_{r}$ of positive radius $r$ centered at $x_{0}$ with

$$
\left.\left(s-h^{\prime}\right)\right|_{K_{r}}>0 .
$$

But this contradicts Proposition 4, since for the harmonic function $h_{0}=1$ and a suitable function $\alpha^{\prime}$ satisfying $\left|\alpha^{\prime}(t)\right| \leq 1$ we would have by (10)

$$
\begin{aligned}
\int_{Z\left(s-h^{\prime}\right)} \alpha^{\prime}+\int_{\bar{B} \backslash Z\left(s-h^{\prime}\right)} \operatorname{sign}\left(s-h^{\prime}\right) & \geq \lambda\left(B_{0}\right)+\lambda\left(K_{r} \cap\left(\bar{B} \backslash \bar{B}_{0}\right)\right)-\lambda\left(\left(\bar{B} \backslash \bar{B}_{0}\right) \backslash K_{r}\right) \\
& \geq \lambda\left(K_{r} \cap\left(\bar{B} \backslash \bar{B}_{0}\right)\right)>0,
\end{aligned}
$$

i.e. $\quad\left(\mathrm{b}_{2}\right)$ of Proposition 4 would be violated, hence $h^{\prime}$ could not be a best $L^{1}$ approximant to $s$, a contradiction.

In order to prove the necessity part of Theorem 2, we establish an auxiliary result concerning the set of zeros of an a.e. strictly subharmonic function.

Lemma 6. Let $D \subset \mathrm{R}^{n}$ be an open set, and let $u$ be a twice differentiable function on $D$ satisfying $\Delta u>0$ a.e. in $D$. Then $\lambda(Z(u))=0$.

For the proof we make use of the notion of points of density of a measurable set $E \subset \mathbf{R}^{n}$ (see Wheeden-Zygmund [20, p. 107]). $x \in E$ is called a point of density of $E$ if

$$
\lim _{\varepsilon \rightarrow 0} \frac{\lambda\left(E \cap Q_{\varepsilon}(x)\right)}{\lambda\left(Q_{\varepsilon}(x)\right)}=1
$$

where $Q_{\varepsilon}(x)$ is the $n$-dimensional cube with edges parallel to the coordinate axes of length $2 \varepsilon$ centered at $x$. Theorem 7.13 by Wheeden-Zygmund [20] states that 
for a measurable set $E \subset \mathbf{R}^{n}$ almost every point of $E$ is a point of density of $E$. Therefore, if we define

$$
E_{d}:=\{x \in E: x \text { is a point of density of } E\},
$$

then we have $\lambda\left(\left(E_{d}\right)_{d}\right)=\lambda\left(E_{d}\right)=\lambda(E)$.

The following lemma will be used for the proof of Lemma 6.

LEMMA 7. Let $D \subset \mathbf{R}^{n}$ be an open set, $V$ an $m$-dimensional normed vector space $(m \in \mathbf{N})$, and $v: D \rightarrow V$ a differentiable function. Then $(Z(v))_{d} \subset Z\left(v^{\prime}\right)$, i.e. for every point of density of $Z(v)$, we have $v^{\prime}(x)=0$.

ProOF. Put $Z_{d}(v):=(Z(v))_{d}$. Without loss of generality, let $V=\mathbf{R}$ (otherwise consider the coordinates $\left.v=\left(v_{1}, \ldots, v_{m}\right)\right)$. In addition, it suffices to prove $v_{x_{1}}:=$ $\partial v / \partial x_{1}=0$ on $Z_{d}(v)$.

Now let $x \in Z_{d}(v)$, and let $\left(\varepsilon_{r}\right)_{r \in \mathbf{N}}$ be a monotone decreasing null sequence of real numbers such that

$$
\frac{\lambda\left(Z(v) \cap Q_{\varepsilon_{r}}(x)\right)}{\lambda\left(Q_{\varepsilon_{r}}(x)\right)} \geq 1-\frac{1}{2^{n} r^{n}}
$$

which is possible by (11). For each $r \geq 2$ define the set

$$
F_{r}:=\left\{x+\sum_{i=1}^{n} a_{i}^{(r)} e_{i}: \frac{\varepsilon_{r}(r-1)}{r} \leq\left|a_{1}^{(r)}\right| \leq \varepsilon_{r},\left|a_{i}^{(r)}\right| \leq \frac{\varepsilon_{r}}{r} \text { for } 2 \leq i \leq n\right\},
$$

where the $e_{i}$ 's are the $n$-dimensional unit vectors. We have

$$
\lambda\left(F_{r}\right)=2^{n} / r^{n} \cdot \varepsilon_{r}^{n}=1 / r^{n} \lambda\left(Q_{\varepsilon_{r}}(x)\right) .
$$

Therefore,

$$
\frac{\lambda\left(F_{r}\right)}{\lambda\left(Q_{\varepsilon_{r}}(x)\right)}=\frac{1}{r^{n}}>\frac{1}{2^{n} r^{n}} .
$$

Hence there exists a $y_{r} \in Z(v) \cap F_{r}$, say $y_{r}=x+\sum_{i=1}^{n} b_{i}^{(r)} e_{i}$. By the differentiablity of $v$, we have

$$
0=v\left(y_{r}\right)-v(x)=v^{\prime}(x)\left(y_{r}-x\right)+o\left(\left|y_{r}-x\right|\right)
$$

for $r \rightarrow \infty$, hence

$$
\lim _{r \rightarrow \infty} \frac{1}{\left|y_{r}-x\right|} \cdot \sum_{i=1}^{n} b_{i}^{(r)} \cdot \frac{\partial v}{\partial x_{i}}(x)=\lim _{r \rightarrow \infty} \frac{v^{\prime}(x)\left(y_{r}-x\right)}{\left|y_{r}-x\right|}=0 .
$$

Now

$$
\lim _{r \rightarrow \infty} \frac{b_{j}^{(r)}}{\left|y_{r}-x\right|}=\lim _{r \rightarrow \infty} \frac{b_{j}^{(r)}}{\sqrt{\sum_{i=1}^{n}\left(b_{i}^{(r)}\right)^{2}}}=\delta_{1 j}
$$

(Kronecker symbol), and this yields $\partial v / \partial x_{1}(x)=0$.

PROOF OF LEMMA 6. Lemma 7 states $u^{\prime}=0$ on $Z_{d}(u)$, and repeating this argument, $u^{\prime \prime}=0$ on $Z_{d}\left(u^{\prime}\right) \supset\left(Z_{d}(u)\right)_{d}$. In particular, we have $\Delta u=0$ on $\left(Z_{d}(u)\right)_{d}$. Hence

$$
0=\lambda\left(\left(Z_{d}(u)\right)_{d}\right)=\lambda\left(Z_{d}(u)\right)=\lambda(Z(u)) \text {. }
$$


PROOF OF THE NECESSITY OF (i) AND (ii). In order to complete the proof of Theorem 2 it remains to show that every best harmonic $L^{1}$-approximant $h^{*} \in$ $\mathscr{H}(B) \cap \mathscr{C}(\bar{B})$ of $s$ satisfies (i) and (ii). Define

$$
\begin{aligned}
E_{-} & :=\left\{x \in \bar{B}:\left(s-h^{*}\right)(x)<0\right\}, \\
E_{0} & :=\left\{x \in \bar{B}:\left(s-h^{*}\right)(x)=0\right\}, \\
E_{+} & :=\left\{x \in \bar{B}:\left(s-h^{*}\right)(x)>0\right\} .
\end{aligned}
$$

Since $S \in \mathscr{C}^{2}(B)$ with $\Delta S>0$ a.e., we have $\Delta\left(s-h^{*}\right)>0$ a.e. (by $h^{*} \in \mathscr{H}(B)$ ). Hence Lemma 6 yields $\lambda\left(E_{0} \cap B\right)=0$, and therefore $\lambda\left(E_{0}\right)=0$. From Proposition $4\left(b_{2}\right)$ we get

$$
\int_{\bar{B}} h \operatorname{sign}\left(s-h^{*}\right)=0 \quad \text { for all } h \in \mathscr{H}(B) \cap \mathscr{C}(\bar{B}) .
$$

From this equality and $\lambda\left(E_{0}\right)=0$ we obtain

$$
\int_{E_{-} \cap B} h=\int_{E_{+} \cap B} h=\frac{1}{2} \int_{\bar{B}} h=\frac{1}{2} \omega_{n} \cdot h(0)
$$

for all $h \in \mathscr{H}(B) \cap \mathscr{C}(\bar{B})$, hence in particular

$$
h(0)=\frac{1}{\lambda\left(E_{-} \cap B\right)} \int_{E_{-} \cap B} h \quad \text { for all } h \in \mathscr{H}\left(\mathbf{R}^{n}\right),
$$

since $\lambda\left(E_{-} \cap B\right)=\frac{1}{2} \omega_{n}$. By virtue of Theorem 1 it remains to show (with $D:=$ $\left.E_{-} \cap B\right)$ that

$\left(\alpha^{\prime}\right) \mathbf{R}^{n} \backslash \overline{\left(E_{-} \cap B\right)}$ is connected, as well as

$\left(\beta^{\prime}\right){\overline{\left(E_{-} \cap B\right)}}^{\circ}=E_{-} \cap B$.

Indeed, if $\left(\alpha^{\prime}\right)$ holds, then (13) and Theorem 1 imply that $\overline{E_{-} \cap B}$ is a closed ball centered at 0 . From $\lambda \overline{\left(E_{-} \cap B\right)}=\frac{1}{2} \lambda(\bar{B})$ we conclude that $\bar{B}_{0}=\overline{E_{-} \cap B}$, therefore, by $\left(\beta^{\prime}\right), B_{0}=E_{-} \cap B$. Hence $s-h^{*}<0$ in $B_{0}$ and $\geq 0$ in $\bar{B} \backslash B_{0}$. By virtue of the continuity of $s-h^{*}$, this implies (i). Now (ii) follows from the fact that $\lambda\left(E_{0}\right)=0$. So it remains to prove $\left(\alpha^{\prime}\right)$ and $\left(\beta^{\prime}\right)$.

$\left(\alpha^{\prime}\right)$ : In order to show the connectedness of $\mathbf{R}^{n} \backslash\left(\overline{E_{-} \cap B}\right)$, we prove that it cannot have a bounded component.

Suppose $X$ were a bounded component of $\mathbf{R}^{n} \backslash\left(\overline{E_{-} \cap B}\right)$. Hence we have $X \subset B$. Then $s-h^{*} \geq 0$ in $X$, thus, by continuity, $\left.\left(s-h^{*}\right)\right|_{\partial X} \geq 0$. We shall show that $\left.\left(s-h^{*}\right)\right|_{\partial X}=0$. Indeed, if $x \in \partial X \cap B$, then $x \in\left(\partial E_{-}\right) \cap B$ (since $X$ is a component of $\left.\mathbf{R}^{n} \backslash\left(\overline{E_{-} \cap B}\right)\right)$, hence $\left(s-h^{*}\right)(x) \leq 0$, therefore $=0$.

For $x \in \partial X \cap \partial B$ we assume indirectly $\left(s-h^{*}\right)(x)>0$. Then there is an open ball $K_{\sigma}(x)$ with $\left(s-h^{*}\right)(y)>0$ for $y \in K_{\sigma}(x) \cap \bar{B}$. Hence $K_{\sigma}(x) \cap\left(\overline{E_{-} \cap B}\right)=\varnothing$. From $K_{\sigma}(x) \cap X \neq \varnothing$ we get that $X$ cannot be a subset of $B$, a contradiction. Therefore, $\left(s-h^{*}\right)(x) \leq 0$, hence again $=0$. This yields $\left.\left(s-h^{*}\right)\right|_{\partial X}=0$. Now the maximum principle implies $s-h^{*}=0$ on $\bar{X}$, thus $X \subset \bar{X} \subset E_{0}$ which contradicts $\lambda\left(E_{0}\right)=0$. This concludes the proof of $\left(\alpha^{\prime}\right)$.

$\left(\beta^{\prime}\right)$ : Since $E_{-} \cap B \subset\left(\overline{E_{-} \cap B}\right)^{\circ}$, it remains to show that $\left(\overline{E_{-} \cap B}\right)^{\circ} \subset E_{-} \cap B$. Let $x \in\left(\overline{E_{-} \cap B}\right)^{\circ}$. Then there is an open ball $K_{\rho}(x)$ of radius $\rho>0$ centered at $x$ with $\overline{K_{\rho}(x)} \subset \overline{E_{-}}$. From this we conclude that $s-h^{*} \leq 0$ in $\overline{K_{\rho}(x)}$. By the maximum principle combined with $\lambda\left(E_{0}\right)=0$ it follows that $s-h^{*}<0$ in $K_{\rho}(x)$, i.e. $x \in E_{-} \cap B$. 
4. Concluding remarks. (1) In a previous paper [10] the special case of Theorem 2 was considered where $n=2$ and where the subharmonic (but not harmonic) function $s$ was supposed to be real analytic in the unit disk, hence $\Delta s>0$ a.e. in $B$. The proof given in [10] does not generalize to dimension $n \geq 3$.

(2) We also should mention a univariate result due to Bernstein [2, p. 330] (see also Lorentz [16, p. 115]) which is related to our approximation problem. Here, however, $n=1$ is considered. Bernstein's theorem reads as follows:

Let $s^{(m)}>0$ in the open interval $]-1,1[$. Then the algebraic polynomial of degree $\leq m-1$ of best approximation to $s$ in the $L^{1}[-1,1]$-norm is the polynomial $q_{m-1}$ which interpolates $s$ in the $m$ points $x_{k}=\cos (k \pi /(m+1)), k=1,2, \ldots, m$.

For the case $m=2$, this result means that the best polynomial approximant $q_{1}$ of degree not exceeding 1 to a convex function $s$ in the $L^{1}[-1,1]$-norm can be computed by interpolation at the points $\left\{-\frac{1}{2}, \frac{1}{2}\right\}$. This result corresponds to our Theorem 2 for the case $n=1$.

(3) Problems of best uniform harmonic approximation were considered by Burchard [4] as well as by Hayman, Kershaw and Lyons [12].

(4) Finally, we show that there exists a function $s \in \mathscr{C}^{2}(B) \cap \mathscr{C}(\bar{B})$, satisfying $\Delta s>0$ a.e. which does not have a best harmonic $L^{1}$-approximant. Indeed, we shall show (in the case $n=3$ ) that for

$$
s(x, y, z)=x^{4} y^{4} z^{4}
$$

condition (ii) of Theorem 2 is violated (see also [10] for a counterexample in the case $n=2$ ). In terms of spherical coordinates

$$
x=r \cdot \sin \theta \cos \varphi, \quad y=r \cdot \sin \theta \sin \varphi, \quad z=r \cdot \cos \theta
$$

we have

$$
s(x, y, z)=: S(r, \theta, \varphi)=\frac{r^{12}}{128} \sin ^{8} \theta \cos ^{4} \theta(\cos 8 \varphi-4 \cos 4 \varphi+3) .
$$

If we define

$$
\begin{aligned}
& S_{1}(r, \theta, \varphi):=\frac{r^{12}}{128} \sin ^{8} \theta \cos ^{4} \theta \cos 8 \varphi \\
& S_{2}(r, \theta, \varphi):=-\frac{4 r^{12}}{128} \sin ^{8} \theta \cos ^{4} \theta \cos 4 \varphi \\
& S_{3}(r, \theta, \varphi):=\frac{3 r^{12}}{128} \sin ^{8} \theta \cos ^{4} \theta
\end{aligned}
$$

then $S=S_{1}+S_{2}+S_{3}$. Since the $S_{i}$ are polynomials, there exist harmonic polynomials $U_{i}$ which solve the Dirichlet problems

$$
U_{i \mid \partial B_{0}}=S_{i \mid \partial B_{0}} \quad \text { for } i=1,2,3 \text {. }
$$

In order to show that condition (ii) of Theorem 2 is violated, it suffices to point out that

$$
\left(S_{3}-U_{3}\right)\left(1, \frac{1}{2} \pi, 0\right)<0 .
$$

Indeed, since $S_{3}-U_{3}$ is independent of $\varphi$, we only have to choose

$$
\varphi= \begin{cases}\pi / 8 & \text { for }\left(S_{1}-U_{1}\right)\left(1, \frac{1}{2} \pi, 0\right) \geq 0 \\ \pi / 4 & \text { for }\left(S_{1}-U_{1}\right)\left(1, \frac{1}{2} \pi, 0\right)<0 \text { and }\left(S_{2}-U_{2}\right)\left(1, \frac{1}{2} \pi, 0\right) \geq 0 \\ 0 & \text { for }\left(S_{1}-U_{1}\right)\left(1, \frac{1}{2} \pi, 0\right)<0 \text { and }\left(S_{2}-U_{2}\right)\left(1, \frac{1}{2} \pi, 0\right)<0 .\end{cases}
$$


Then $\left(S_{i}-U_{i}\right)\left(1, \frac{1}{2} \pi, \varphi\right) \leq 0$ for $i=1,2$, hence from (15)

$$
(S-U)\left(1, \frac{1}{2} \pi, \varphi\right)<0,
$$

where $U:=U_{1}+U_{2}+U_{3}$. By continuity of $S-U$ it follows that condition (ii) of Theorem 2 is not satisfied.

So it remains to prove (15). We expand $S_{3}$ in terms of Legendre polynomials (normed by $P_{n}(1)=1$; for the coefficients occurring see $[1$, p. 798])

$$
\begin{aligned}
S_{3}(r, \theta, \varphi)=r^{12}\left\{\frac{24}{676039} P_{12}(\cos \theta)-\frac{12}{81719} P_{10}(\cos \theta)+\frac{3}{25935} P_{8}(\cos \theta)\right. \\
\left.+\frac{36}{124355} P_{6}(\cos \theta)-\frac{6}{12155} P_{4}(\cos \theta)+\frac{1}{5005}\right\} .
\end{aligned}
$$

Since any function of the form $r^{k} P_{k}(\cos \theta)$ is harmonic (see e.g. Hobson $[14, \mathrm{p}$. 123]), the solution of the Dirichlet problem (14) for $i=3$ is given by

$$
\begin{aligned}
U_{3}(r, \theta, \varphi)= & \frac{24 r^{12}}{676039} P_{12}(\cos \theta)-\frac{1}{\sqrt[3]{4}} \cdot \frac{12 r^{10}}{81719} P_{10}(\cos \theta) \\
& +\frac{1}{2 \sqrt[3]{2}} \cdot \frac{3 r^{8}}{25935} P_{8}(\cos \theta)+\frac{1}{4} \cdot \frac{36 r^{6}}{124355} P_{6}(\cos \theta) \\
& -\frac{1}{4 \sqrt[3]{4}} \cdot \frac{6 r^{4}}{12155} P_{4}(\cos \theta)+\frac{1}{16} \cdot \frac{1}{5005} .
\end{aligned}
$$

Using $P_{10}(0)=-63 / 256, P_{8}(0)=35 / 128, P_{6}(0)=-5 / 16$, and $P_{4}(0)=3 / 8$, we compute

$$
\begin{aligned}
\left(S_{3}-U_{3}\right)\left(1, \frac{1}{2} \pi, 0\right)= & -\frac{12}{81719}\left(1-\frac{1}{\sqrt[3]{4}}\right) \cdot\left(-\frac{63}{256}\right) \\
& +\frac{3}{25935}\left(1-\frac{1}{2 \sqrt[3]{2}}\right) \cdot \frac{35}{128}+\frac{36}{124355}\left(1-\frac{1}{4}\right)\left(-\frac{5}{16}\right) \\
& -\frac{6}{12155}\left(1-\frac{1}{4 \sqrt[3]{4}}\right) \cdot \frac{3}{8}+\left(1-\frac{1}{16}\right) \cdot \frac{1}{5005} \\
< & -4 \cdot 10^{-6} .
\end{aligned}
$$

Thus (15) is verified, hence $s(x, y, z)=x^{4} y^{4} z^{4}$ does not possess a best harmonic $L^{1}$-approximant on the unit ball in $\mathbf{R}^{3}$ with respect to $\mathscr{H}(B) \cap \mathscr{C}(\bar{B})$.

This example also shows that condition (i) of Theorem 2 does not imply condition (ii).

\section{REFERENCES}

1. M. Abramowitz and I. A. Stegun, eds., Handbook of mathematical functions, Dover, New York, 1972.

2. S. N. Bernstein, Collected works, Vol. 1, Izdat. Akad. Nauk SSSR, Moscow, 1952.

3. M. Brelot, Sur l'approximation et la convergence dans la théorie des fonctions harmoniques ou holomorphes, Bull. Soc. Math. France 73 (1945), 55-70.

4. H. G. Burchard, Best uniform harmonic approximation, Approximation Theory II (G. G. Lorentz, C. K. Chui, L. L. Schumaker, eds.), Academic Press, New York and London, 1976, pp. 309-314.

5. E. W. Cheney, Introduction to approximation theory, McGraw-Hill, New York, 1966. 
6. J. Deny, Systèmes totaux de fonctions harmoniques, Ann. Inst. Fourier (Grenoble) 1 (1949), 103-113.

7. T. W. Gamelin, Uniform algebras, Prentice-Hall, Englewood Cliffs, N.J., 1969.

8. P. M. Gauthier, M. Goldstein and W. H. Ow, Uniform approximation on closed sets by harmonic functions with Newtonian singularities, J. London Math. Soc. (2) 28 (1983), 71-82.

9. D. Gilbarg and N. S. Trudinger, Elliptic partial differential equations of second order, $\$$ inger, Berlin, Heidelberg and New York, 1977.

10. M. Goldstein, W. Haussmann and K. Jetter, Best harmonic $L^{1}$ approximation to subharmonic functions, J. London Math. Soc. (2) 30 (1984), 257-264.

11. W. K. Hayman and P. B. Kennedy, Subharmonic functions, Academic Press, London and New York, 1976.

12. W. K. Hayman, D. Kershaw and T. J. Lyons, The best harmonic approximant to a continuous function, Anniversary Volume on Approximation Theory and Functional Analysis (P. L. Butzer, R. L. Stens, B. Sz.-Nagy, eds.), Internat. Ser. Numer. Math. 65, Birkhäuser, Basel, Boston and Stuttgart, 1984, pp. 317-327.

13. L. I. Hedberg, Approximation in the mean by solutions of elliptic equations, Duke Math. J. 40 (1973), 9-16.

14. E. W. Hobson, The theory of spherical and ellipsoidal harmonics, Chelsea, New York, 1965.

15. Ü. Kuran, On the mean value property of harmonic functions, Bull. London Math. Soc. 4 (1972), 311-312.

16. G. G. Lorentz, Approximation of functions, Holt, Rinehart and Winston, New York, 1966.

17. J. C. Polking, Approximation in $L^{p}$ by solutions of elliptic partial differential equations, Amer. J. Math. 94 (1972), 1231-1244.

18. J. R. Rice, The approximation of functions, Vol. 1, Addison-Wesley, Reading, Mass., 1964.

19. I. Singer, Best approximation in normed linear spaces by elements of linear subspaces, Springer, Berlin, Heidelberg and New York, 1970.

20. R. L. Wheeden and A. Zygmund, Measure and integral: An introduction to real analysis, Dekker, New York and Basel, 1977.

Department of Mathematics, Arizona State University, Tempe, Arizona 85281 (Current address of Myron Goldstein)

Department of Pure Mathematics, The University of LiVerpool, LiVerpool L69 3BX, ENGLAND

Department of MAThematics, UNIVERSity OF DUiSBURG, D-4100 Duisburg, West GERMANY (Current address of Werner Haussmann and Lothar Rogge) 